\title{
Evidence for Nonradiative Energy Transfer in Graphene-Oxide-Based Hybrid Structures
}

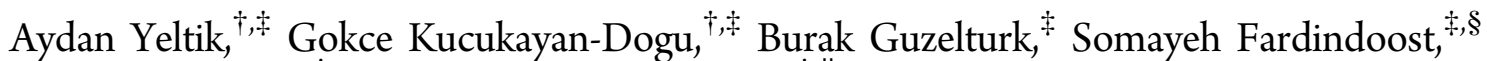

Yusuf Kelestemur, and Hilmi Volkan Demir ${ }^{*,+, \|,}$

${ }^{\ddagger}$ Department of Physics, Department of Electrical and Electronics Engineering, and UNAM - Institute of Materials Science and Nanotechnology, Bilkent University, Ankara, Turkey, 06800

${ }^{\S}$ Institute for Nanoscience and Nanotechnology, Sharif University of Technology, Tehran, Iran14588

"LUMINOUS! Center of Excellence for Semiconductor Lighting and Displays, Microelectronics Division, School of Electrical and Electronics Engineering, and Physics and Applied Physics Division, School of Physical and Mathematical Sciences, Nanyang

Technological University, Nanyang Avenue, Singapore, 639798

\section{Supporting Information}

ABSTRACT: Solution processed graphene variants including graphene oxide (GO) and reduced graphene oxide (RGO) are promising materials for potential optoelectronic applications. To date, efficiency of the excitation energy transfer into GO and RGO thin layers has not been investigated in terms of donor-acceptor separation distance. In the present work, we study nonradiative energy transfer (NRET) from CdSe/CdS quantum dots into single and/or double layer GO or RGO using time-resolved fluorescence spectroscopy. We observe shorter lifetimes as the separation distance between the QDs and GO or RGO decreases. In accordance with these lifetimes,
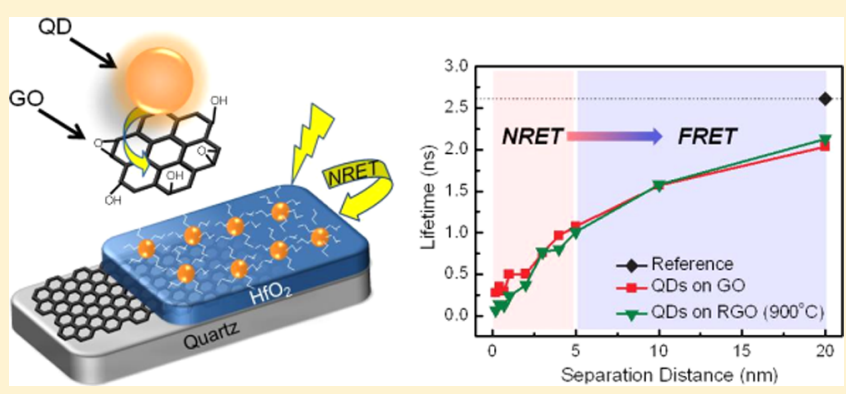
the rates reveal the presence of two different mechanisms dominating the NRET. Here we show that excitonic NRET is predominant at longer intervals while both excitonic and nonexcitonic NRET exist at shorter distances. In addition, we find the NRET rate behavior to be strongly dependent on the reduction degree of the GO-based layers. We obtain high NRET efficiency levels of $\sim 97$ and $\sim 89 \%$ for the closest separation of the QD-RGO pair and the QD-GO pair, respectively. These results indicate that strong NRET from QDs into thin layer GO and RGO makes these solution-processable thin films promising candidates for light harvesting and detection systems.

\section{INTRODUCTION}

Graphene, two-dimensional honeycomb structure of carbon atoms, has become a subject of great interest due to its unique optical and electronic properties. ${ }^{1}$ Despite its one-atom-thick nature, optical absorbance of graphene is $\sim 2.3 \%$ over a broad photonic energy spectrum above $0.5 \mathrm{eV}$ (significantly in visible and infrared region), which originates from its linear energy dispersion near the Fermi level and its zero band gap structure. ${ }^{2,3}$ As compared to other materials, graphene provides significantly large surface area $\left(2630 \mathrm{~m}^{2} \mathrm{~g}^{-1}\right)^{4}$ and high intrinsic room-temperature mobility (up to $250000 \mathrm{~cm}^{2} \mathrm{~V}^{-1} \mathrm{~s}^{-1}$ ). ${ }^{5}$ These properties make graphene promising for optoelectronic applications. ${ }^{6}$ Additionally, graphene is shown to be a good exciton (electron-hole pair) sink due to highly efficient nonradiative energy transfer (NRET) from the near-by fluorescent nanostructures through dipole-dipole coupling, which is also known as Förster-type resonant energy transfer (FRET) $)^{7,8}$ For this type of hybrid structure, including a donor and an acceptor, nonexcitonic NRET mechanisms are also possible at short ranges feeding higher excitation energy into the acceptor. ${ }^{9,10}$ Thus, utilization of graphene as an efficient excitation energy acceptor through NRET holds promise for various photonic and optoelectronic applications including new ways of light harvesting based on graphene's hybrid structures. $^{11,12}$

Previous reports present that graphene accepts excitons from hybridized quantum confined structures (e.g., quantum dots (QDs)) and dye molecules. ${ }^{7,8}$ This exciton transfer has been experimentally and theoretically demonstrated to be dominantly FRET. ${ }^{7,8,13,14}$ In this regard, owing to their favorable optical properties such as size tunable and strong luminescence emission, and photostability, QDs are good candidates to sensitize graphene for possible application in photodetectors and light harvesting systems. ${ }^{15}$ To date, although epitaxial graphene has been widely studied solution processed graphene, that is, graphene oxide (GO) and reduced graphene oxide (RGO), can offer unique capabilities including ease of processing at low cost, large area coverage, and tunable energy

Received: August 23, 2013

Revised: November 10, 2013

Published: November 13, 2013 
(a)

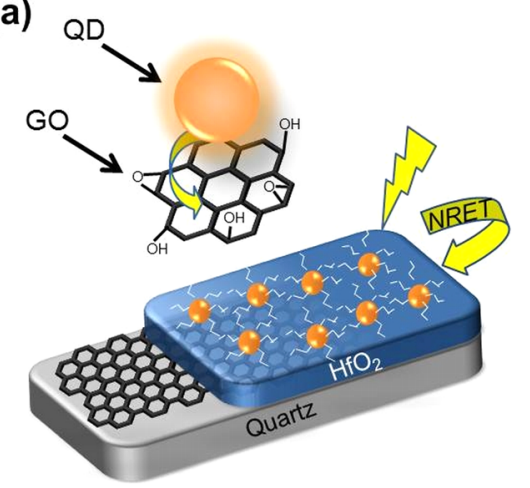

(b)

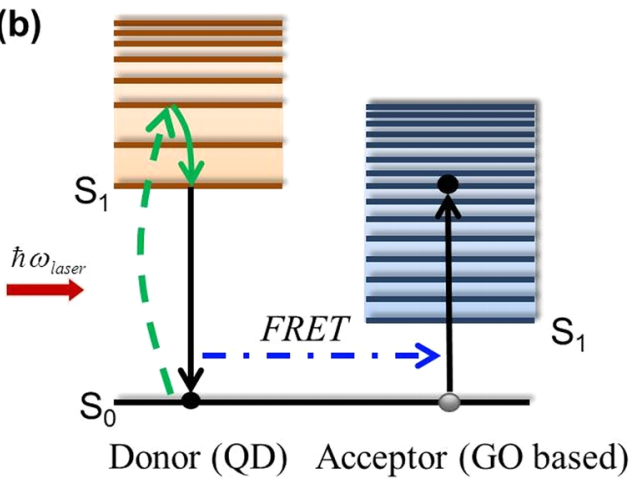

Figure 1. (a) Schematic representation of the hybrid $\mathrm{QD} / \mathrm{HfO}_{2} / \mathrm{GO}$ structure. (b) Energy diagram for the FRET from QD into GO-based acceptor.

gap, which cannot be satisfied by the epitaxial graphene. ${ }^{16,17}$ Therefore, NRET into GO or RGO considering both FRET and nonexcitonic NRET processes, which may exhibit an energy quenching range comparable to graphene, is particularly important to explore and it is essential to understand feasibility and extend of excitation energy sink as well as the required conditions. $^{8,13,14,18}$

Recently, most of the experimental studies have highlighted the importance of fluorescence quenching efficiency in QDs$\mathrm{GO}^{19}$ and dye-RGO ${ }^{18}$ hybrids for the development of various application fields. In these studies, fluorescence quenching measurements provide limited information about the energy transfer mechanism. For the detailed analysis of excited state dynamics of the fluorophores, time-resolved fluorescence spectroscopy (TRF) is required. As a powerful technique, TRF can reveal the fluorescence lifetime modifications, which can be directly attributed to the NRET since it opens up a new channel for the donor excitons to relax. TRF decay rates enable the analysis of NRET channels between QDs and GO or RGO, which occurs under a broad resonance absorption spectrum at short separations as compared to the ranges of radiative energy transfer (RET). In general, NRET efficiency is strongly dependent on separating distance between the donor and the acceptor. ${ }^{20,21}$ However, to date, the efficiency of the NRET into GO or RGO has not been known in terms of the donoracceptor distance.

Herein, we report the distance dependence of the NRET in QD-GO and QD-RGO hybrid films, where a thin layer of QD coating is achieved over uniformly full-covered thin GO (1-2 layers) and thermally annealed thin RGO layers. Furthermore, NRET rates are found to be dependent on the degree of reduction of GO films. The NRET efficiencies are measured to be around 97 and $89 \%$ at the closest separation for the QDRGO and the QD-GO hybrids, respectively. These results indicate that the strong NRET from QDs into GO-based thin layers makes these hybrid structures promising candidates to be used in light harvesting and detecting applications.

\section{EXPERIMENTAL SECTION}

Preparation of GO Solution. For the preparation of welldispersed GO solution, the GO powder (Timesnano, Chengdu Organic Chemicals Co. Ltd., China) was first suspended in deionized water at a concentration of $1.6 \mathrm{mg} / \mathrm{mL}$ and then ultrasonicated for $30 \mathrm{~min}$. Subsequently, the prepared solution was centrifuged at $14500 \mathrm{rpm}$ for $10 \mathrm{~min}$. After the centrifugation, multiple GO layers were descended and the supernatant is recovered for resonication and recentrifugation in the same manner. The resultant supernatant solution, which is stable against precipitation, was the GO solution used in this study.

Coating of GO Thin Films. The $3 \mathrm{~mm}$ thick $1.5 \times 1.5 \mathrm{~cm}^{2}$ sized quartz slides were used as the substrates for GO coating. Prior to the GO coating, quartz substrates were cleaned in piranha solution (7:3 vol $\mathrm{H}_{2} \mathrm{SO}_{4} / \mathrm{H}_{2} \mathrm{O}_{2}$ mixture) for $40 \mathrm{~min}$, rinsed with deionized water, and dried under nitrogen flow. Then, the prepared GO solution was spin-coated over the quartz substrates at $2000 \mathrm{rpm}$ for $3 \mathrm{~min}$, which resulted in wellcovered thin GO films.

Thermal Reduction of GO Thin Films. GO coated quartz wafers were placed in a vacuum capable quartz-tube furnace with controlled pressure. Substrates were heated up to 625 or $900{ }^{\circ} \mathrm{C}$ and held at these temperatures for $30 \mathrm{~min}$ under flowing $\mathrm{Ar} / \mathrm{H}_{2}(1: 1)$ atmosphere. Here, the heating rate is a key parameter for the thermal reduction process to accomplish the reduction of GO without any film loss due to the presence of oxygen containing groups. Therefore, low heating rate $\left(5^{\circ} \mathrm{C} /\right.$ min) was preferred to eliminate any possible film loss from substrates. The prepared GO and RGO films were characterized by using Raman spectroscopy at a laser wavelength of $532 \mathrm{~nm}, \mathrm{X}$-ray photoelectron spectroscopy (XPS), and tapping mode atomic force microscopy (AFM).

Deposition of $\mathrm{HfO}_{2}$ and QD Layers. For the systematic investigation of the separation dependence of energy transfer from QDs into GO and RGO, we coated a thin layer of $\mathrm{HfO}_{2}$ on the GO and RGO films using atomic layer deposition (ALD) at a deposition temperature of $150{ }^{\circ} \mathrm{C}$. In this study, $0.2,0.4,0.7,1.0,2.0,3.0,4.0,5.0,10.0$, and $20.0 \mathrm{~nm}$ thick $\mathrm{HfO}_{2}$ layers were used as the barrier layer. To determine the roughness of $\mathrm{HfO}_{2}$ layers on GO and RGO films, we obtained AFM images as represented in Figure $S 1$ (Supporting Information), and found the roughness of the GO layer to be around $0.8 \mathrm{~nm}$ and the roughness of RGO layer to be around $0.4 \mathrm{~nm}$. The roughness of GO and RGO films was found not to change with the ALD coating of thin $\mathrm{HfO}_{2}$ layers whose film thicknesses are $0.2,0.4$, and $0.7 \mathrm{~nm}$. Variation in the roughness as compared to the GO and RGO films was found to become $0.3 \mathrm{~nm}$ for the samples coated with thicker $\mathrm{HfO}_{2}$ layers with thicknesses of 1.0, 2.0, 3.0, 4.0, 5.0, 10.0, and $20.0 \mathrm{~nm}$. All these AFM results indicate that roughness is low and is expected not to have a significant impact on the energy transfer in our structures. CdSe/CdS QDs were spin-coated on the GO and RGO samples (with/without $\mathrm{HfO}_{2}$ ) at $2000 \mathrm{rpm}$ for $180 \mathrm{~s}$. The average diameter of the QDs was measured to be around 5.8 $\mathrm{nm}$ by high resolution transmission electron microscopy (HR- 


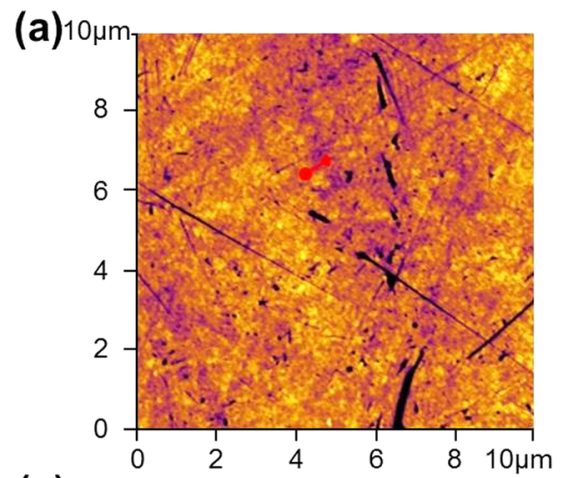

(c)

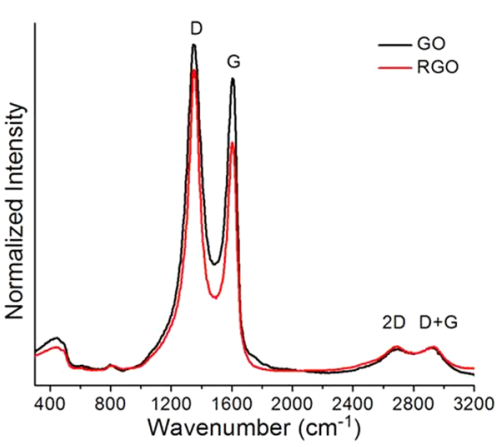

(e)

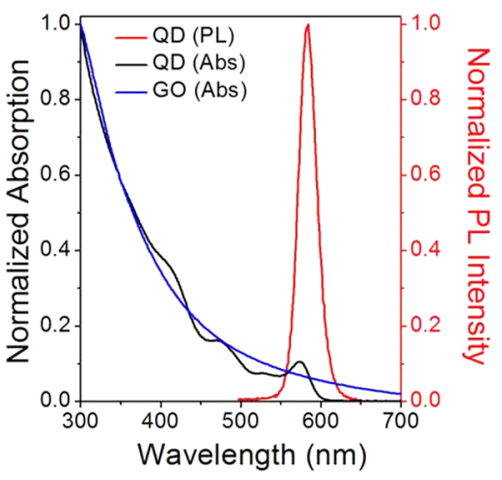

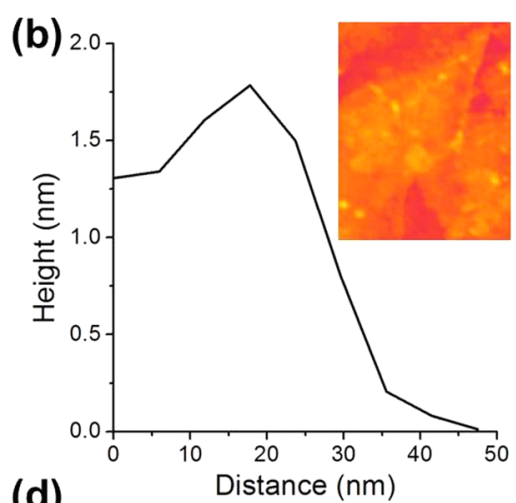

(d)

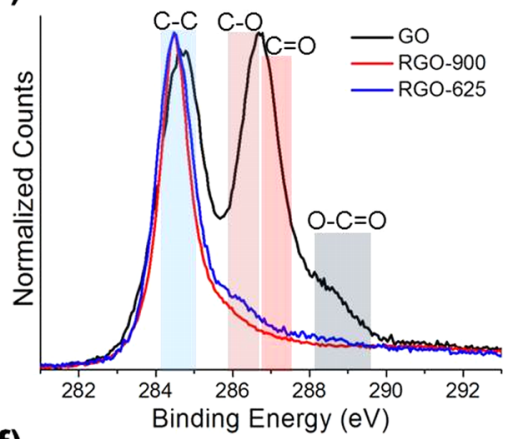

(f)

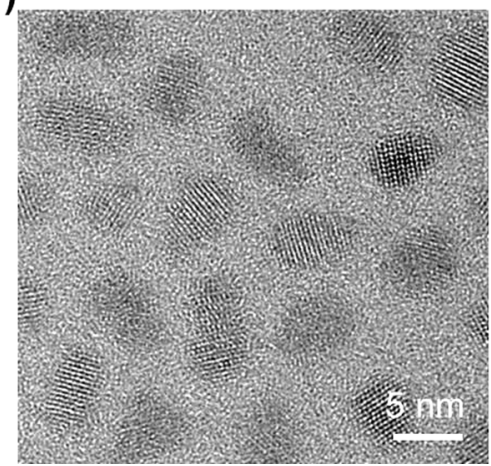

Figure 2. (a) Tapping mode AFM image of the GO thin film on quartz. (b) Height profile along the red line presented in (a). Inset shows the AFM image of an isolated single GO sheet on quartz. (c) Raman spectra of GO and RGO thin film reduced at $900{ }^{\circ} \mathrm{C}$. (d) XPS spectra of GO and RGO thin films reduced at 625 and $900{ }^{\circ} \mathrm{C}$. (e) Normalized absorption (black line) and PL (red line) spectra of CdSe/CdS QDs with normalized absorption of GO solution (blue line). (f) HR-TEM image of the QDs used in the study.

TEM). The thicknesses of $\mathrm{HfO}_{2}$ and $\mathrm{QD}$ layers were determined by ellipsometry. From the analysis of the ellipsometry data for the $\mathrm{HfO}_{2}$ layers, we observed small mean square errors in film thickness determination less than $0.02 \mathrm{~nm}$.

TRF Spectroscopy of QDs-GO and QDs-RGO Hybrid Structures. QDs-GO and QDs-RGO hybrid structures both with and without the $\mathrm{HfO}_{2}$ layer were characterized by TRF in order to investigate the energy transfer mechanism between the QDs and GO or RGO layers. Room-temperature fluorescence decays of the QDs were recorded in vacuum to completely eliminate the photo-oxidation of the QDs. The laser excitation of the TRF system was performed at $375 \mathrm{~nm}$. The TRF detection wavelength was set to $580 \mathrm{~nm}$, which is the peak emission wavelength of the QDs on GO or RGO films.

\section{RESULTS AND DISCUSSION}

NRET mechanism was investigated on the hybrid structures composed of CdSe/CdS QDs decorated on top of $\mathrm{HfO}_{2}$ deposited GO or RGO films which serve as the donor, the separation dielectric layer and the acceptor, respectively. The schematic representation of the QD-GO-based structures is shown in Figure 1a. The $\mathrm{HfO}_{2}$ thickness is carefully set to 0.2, $0.4,0.7,1.0,2.0,3.0,4.0,5.0,10.0$, and $20.0 \mathrm{~nm}$ on GO- or RGO-coated quartz substrates through ALD. These thicknesses are much smaller than the emission wavelength of the QDs (580 nm in film), which is a favorable condition for the occurrence of NRET before RET becomes the dominant transfer mechanism. ${ }^{8}$ NRET refers to the combination of the excitonic transfer, which is based on FRET in our study (Figure 1b) and the nonexcitonic transfer coming from the contribution of charge transfer mechanisms.

Figure 2a shows the AFM image of the $10 \times 10 \mu \mathrm{m}^{2}$ area of the spin-coated GO film on a quartz substrate. It is clear that GO coating on the surface is continuous having an average roughness (rms) of $1.3 \mathrm{~nm}$, which is comparable to the average roughness of only quartz. From the height profile analysis as presented by the red line in Figure 2a, the average thickness of the GO film is found to be $\sim 2 \mathrm{~nm}$ (Figure 2b), which is also verified via high-sensitivity optical profilometry technique 

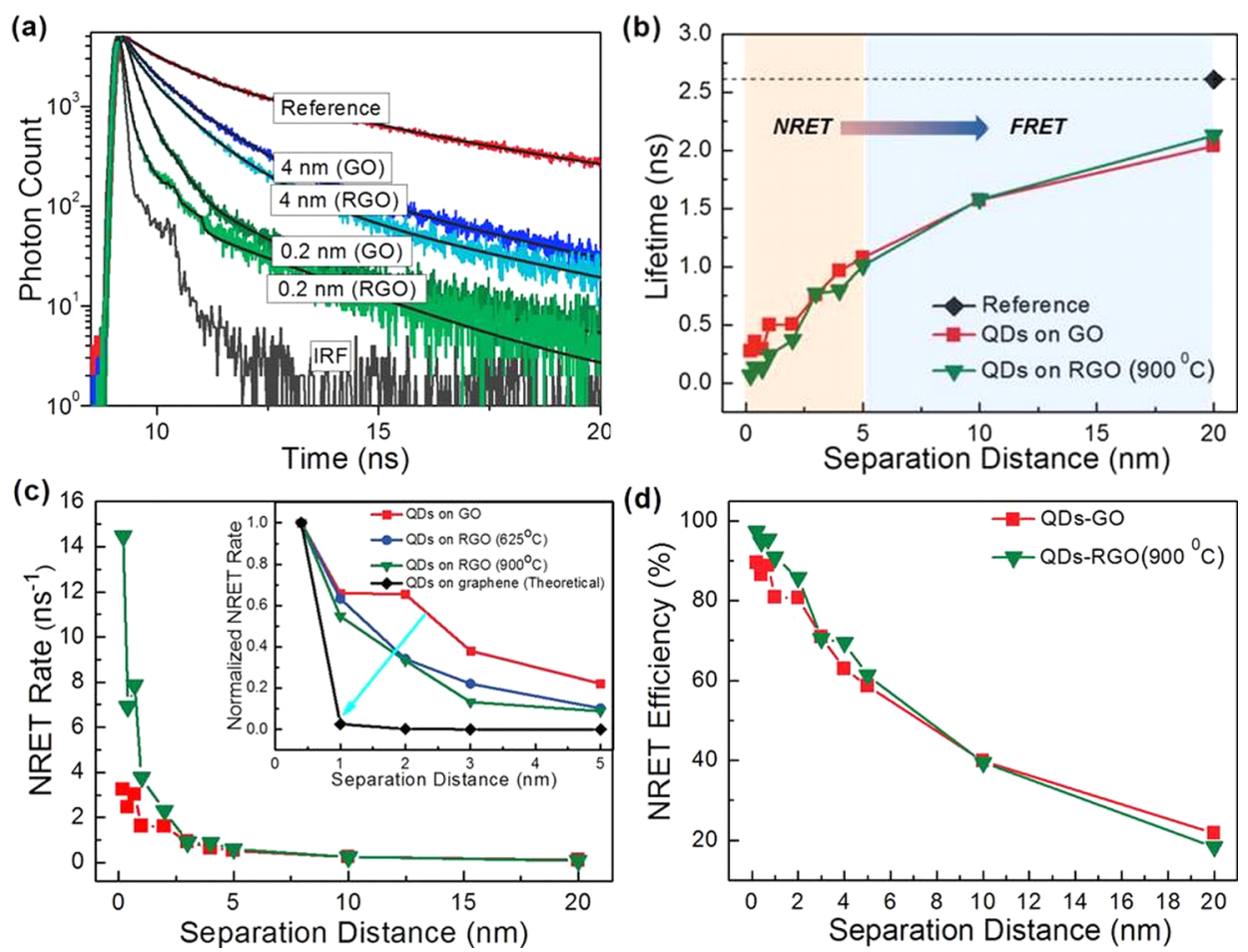

Figure 3. (a) Experimental TRF decays of the QDs on GO and RGO thin films on quartz for the $\mathrm{HfO}_{2}$ separation distances of 4.0 and $0.2 \mathrm{~nm}$, and $20.0 \mathrm{~nm}$ for the QDs without GO and RGO at the bottom (reference). Solid black lines are multiexponential fits to the data, and gray decay curve is the laser diode response function (IRF). (b) TRF lifetimes of the QDs as a function of the distance from GO and RGO; and of the reference. (c) Separation distance dependence of the NRET rates of the QDs on GO and RGO reduced at $900{ }^{\circ} \mathrm{C}$. Inset shows the experimental decays of the QDs on GO, RGO reduced at $625^{\circ} \mathrm{C}$, RGO reduced at $900^{\circ} \mathrm{C}$ and the theoretical decay of the QDs on graphene (d) NRET efficiency of the QDs on GO and RGO reduced at $900{ }^{\circ} \mathrm{C}$ as a function of the separation distance.

(Zygo 3D Optical Profilometer, U.S.A.). Isolated GO sheets obtained through spin coating of lower density GO solution lead to a film thickness of $\sim 1 \mathrm{~nm}$ (Figure $2 \mathrm{~b}$, inset), and such a film thickness value is larger than a single layer pristine graphene owing to the presence of oxygen-containing groups in the GO structure. ${ }^{16}$ Therefore, GO samples have on the average double equivalent layers of GO sheets on the quartz substrates (AFM image given in the inset in Figure 2b).

Thermal reduction at various temperatures is employed to obtain RGO under $\mathrm{H}_{2} / \mathrm{Ar}$ ambient in an atmosphere-controlled furnace. To evaluate the effect of reduction process on film characteristics, GO and RGO films were analyzed by using Raman spectroscopy and XPS. Raman spectroscopy is a powerful technique for the analysis of defects and disorders in carbon-based materials. ${ }^{22}$ For typical GO and RGO films, there are two characteristic peaks located around $1350 \mathrm{~cm}^{-1}$ (D band) and $1580 \mathrm{~cm}^{-1}$ ( $\mathrm{G}$ band), which are assigned to the disordered carbon atoms and the vibration of $\mathrm{sp}^{2}$ bonded carbon atoms, respectively. ${ }^{23,24}$ In this study, D and G bands appear at the wavenumbers of 1352 and $1605 \mathrm{~cm}^{-1}$ for both prepared GO and RGO films, which is in good agreement with the previous studies (Figure 2c). ${ }^{23,24}$ Although some of the recent reports show that no shift occurs in D and G peaks after reduction, ${ }^{25-27}$ there are few studies reporting the red shift of the G band. ${ }^{18,28}$ In our case, we did not observe any shift for D or $G$ peaks after the reduction process while $D$ band was narrowed owing to the loss of oxygen groups. Furthermore, the intensity ratio $I(D) / I(G)$ is used to characterize the quality of GO and RGO films. ${ }^{28,29}$ The $I(D) / I(G)$ intensity ratio for the GO film is found to be 1.1, which is increased to 1.3 after the reduction. There is a conflict in the literature for the effect of reduction on $I(G) / I(D)$ ratio: while some reports claim no change or a decrease for the ratio of $I(G) / I(D),{ }^{28,30,31}$ others observe an increase. ${ }^{26,32,33}$ The possible reason of the increase in the ratio in our study might be attributed to the presence of unrepaired defects after the removal of oxygen groups with the help of reduction at the specified temperatures. ${ }^{26,33}$

Furthermore, XPS analysis was conducted on the GO and RGO films to better understand the chemical structure after the reduction process for the NRET study. Figure $2 \mathrm{~d}$ shows the $\mathrm{C} 1$ s peak for GO and RGO films reduced at two different temperatures, 625 and $900{ }^{\circ} \mathrm{C}$. Deconvolution of $\mathrm{C} 1 \mathrm{~s}$ peak for GO clearly indicates the presence of $\mathrm{C}-\mathrm{C}$ bond $(\sim 284.8 \mathrm{eV})$ beside the $\mathrm{C}-\mathrm{O}(\sim 286.3 \mathrm{eV}), \mathrm{C}=\mathrm{O}(\sim 287.6 \mathrm{eV})$, and $\mathrm{O}-$ $\mathrm{C}=\mathrm{O}(\sim 289.0 \mathrm{eV})$ bonds, which is reasonably consistent with the previous reports for GO films. ${ }^{34-36}$ However, for the case of RGO, the main C-C peak chemically shifts to $284.3 \mathrm{eV}$ and becomes narrower than the peak at GO case. Furthermore, the $\mathrm{C} / \mathrm{O}$ atomic ratio was obtained through the deconvolution of $\mathrm{C}$ 1s peaks for GO and RGO samples. The C/O ratio of GO film was found to be 0.9 , which was increased to 2.7 and 3.1 after the reduction at 625 and $900{ }^{\circ} \mathrm{C}$, respectively. All these $\mathrm{C} 1 \mathrm{~s}$ peak properties of the RGO samples indicate that there is a significant amount of reduction on the GO films at $900{ }^{\circ} \mathrm{C}$ by considering the $\mathrm{C} / \mathrm{O}$ ratio and the decrease in the intensity of oxygenated carbon peaks.

Figure 2e shows the optical absorbance and fluorescence of the $5.8 \mathrm{~nm}$ sized CdSe/CdS QDs along with the absorbance of the GO solution in water. QDs exhibit PL centered at $~ 590$ $\mathrm{nm}$. Owing to the narrow size distribution of the QDs, higher order excitonic peaks are also visible in the UV-vis absorbance. For NRET, a spectral overlap is required between emission of 
the donor and absorption of the acceptor. As shown in Figure $2 \mathrm{e}$, there is a considerable absorption by GO in the emission range of the QDs. Beside PL analysis of the size distribution HR-TEM analysis provides evidence for a narrow size distribution of the QDs (Figure 2f).

After the preparation of $\mathrm{GO}$ and RGO surfaces, $\mathrm{HfO}_{2}$ layer is deposited on the GO and RGO films by ALD technique in order to systematically investigate the effect of separation layer thickness on NRET from the donor QDs to the acceptor GO or RGO films. First, TRF was employed to measure the fluorescence decays of the QD emission when placed on top of GO or RGO films having varying $\mathrm{HfO}_{2}$ thickness. Figure 3a depicts the exemplary room-temperature TRF decay curves with the corresponding numerical fits for the QD-GO and QDRGO hybrid structures having 4.0 and $0.2 \mathrm{~nm}$ thick $\mathrm{HfO}_{2}$ layer as the separation layer between the QDs and GO or RGO thin film on quartz substrates in addition to the instrument response function (IRF) of the TRF system. The spin-coated QDs on quartz that was predeposited with a $20.0 \mathrm{~nm}$ thick $\mathrm{HfO}_{2}$ film is used as a reference. Here, the $\mathrm{HfO}_{2}$ predeposition is used to ensure the QDs film quality. The fluorescence lifetimes of the QDs were fitted via multiexponential fitting and the average lifetimes were calculated using amplitude averaging technique (reduced $\chi^{2} \sim 1$ ). In comparison with the reference sample, there was a significant alteration of the QDs emission decay kinetics as the separation distance between the QDs and the GO-based films was decreased. Clear reduction in lifetimes with the decreasing distance indicates faster decays at shorter separations (Figure 3a). In Figure 3b, fluorescence lifetimes of the QDs are depicted for the QD-GO, QD-RGO, and QDquartz structures parametrized with respect to $\mathrm{HfO}_{2}$ separation layer thickness. The reference QD lifetime was found to be 2.61 ns, which was verified to be independent from the thickness of $\mathrm{HfO}_{2}$ deposited on quartz substrate. The lifetimes of QD-GO and QD-RGO (reduced at $900{ }^{\circ} \mathrm{C}$ ) structures prolong as the separation distances increase. At longer distances $(>5 \mathrm{~nm}$ ), the QDs attain almost the same lifetime values for both GO and RGO acceptors. However, for the shorter $\mathrm{HfO}_{2}$ separation distances $(<5 \mathrm{~nm})$, lifetime of the QDs on top of RGO is shorter than that on GO. Two different lifetime behaviors with respect to distances indicate the presence of more than one mechanisms acting simultaneously, which can be explained by the combination of FRET and nonexcitonic NRET. To better understand the underpinning mechanisms, the excitation transfer rates are calculated using the expression $\gamma_{\mathrm{NRET}}=\gamma_{\mathrm{DA}}$ $-\gamma_{\mathrm{D}}$, where $\gamma_{\mathrm{DA}}\left(1 / \tau_{\mathrm{DA}}\right)$ is the rate for the donor-acceptor hybrid structure (QDs-GO or RGO) and $\gamma_{\mathrm{D}}\left(1 / \tau_{\mathrm{D}}\right)$ is the rate for the structure with only donor (QDs). Figure $3 \mathrm{c}$ presents the NRET rates as a function of the $\mathrm{HfO}_{2}$ separation thickness. In accordance with the lifetimes shown in Figure $3 b$, at the shorter separations, the rates are modified by the combination of both FRET and nonexcitonic NRET. For the longer separations, the rates are dominated by FRET since the charge transfer processes are strongly distance limited.

Previously, it was debatable whether the observed extraordinary steady-state PL quenching of the fluorescence of the emitters in the proximity of graphene-based film is due to the FRET or nonexcitonic NRET. The parametric study enables us to determine that both types of mechanisms are viable (Figure $3 \mathrm{~b}, \mathrm{c})$. In a recent study, it has been demonstrated that different $\mathrm{sp}^{2} / \mathrm{sp}^{3}$ ratios in the functionalized and as-synthesized carbon nanotubes alter the quenching of the fluorescent molecules possibly due to varying nonexcitonic NRET rates. ${ }^{37}$ In our case,
RGO is shown to exhibit greater effect on the lifetimes of the QDs in the nonexcitonic NRET regime. This is expected since GO is decorated with epoxy and hydroxyl groups in its hexagonal basal plane and, following the reduction process, the density of oxygen containing groups is decreased. As a result, it leads to larger $\mathrm{sp}^{2} / \mathrm{sp}^{3}$ ratio in $\mathrm{RGO}$ as compared to that in $\mathrm{GO}$, which would favor the nonexcitonic NRET.

Recently, there have been a number of studies reporting the NRET rates for graphene-based hybrid structures with the inverse fourth order power of the separation distance (1/ $\left.\mathrm{d}^{4}\right) \cdot{ }^{8,14}$ However, there is no study for the reduction dependent systematic rate analysis of GO-based hybrids although there are reports for the investigation of energy transfer into these structures. ${ }^{19,38}$ Using this dependency, a theoretical distance dependence NRET rate for a graphene layer is represented by a black line in the inset of Figure 3c. In order to investigate the effect of reduction on the energy transfer rate behavior, the normalized NRET rates of GO and RGO samples reduced at either 625 or $900^{\circ} \mathrm{C}$ are also presented as an inset in Figure 3c. It is clear that there is an increase in the order of inverse power with the temperature of the reduction process and it approaches to that of graphene, which has fourth order dependence.

Furthermore, we calculated the NRET efficiencies using the relation $\eta=\gamma_{\mathrm{NRET}} /\left(\gamma_{\mathrm{NRET}}+\gamma_{\mathrm{D}}\right)$. High efficiencies were obtained, yielding around $97 \%$ for the closest separation of QDs-RGO $\left(900^{\circ} \mathrm{C}\right)$ structures and around $89 \%$ for the closest separation of QDs-GO structures, as depicted in Figure 3d. It is also worth noting again that efficiencies become sampleindependent as the separation between the donor and the acceptor increases.

\section{CONCLUSION}

We investigated NRET from CdSe/CdS QDs into uniformly coated GO and RGO thin layers using TRF spectroscopy. It is shown that, as the separation distance between the QDs and GO or RGO decreases, TRF lifetimes are shortened, which indicates faster NRET rates. For both QDs-GO and QDs-RGO hybrids, NRET mechanism consists of excitonic-NRET at longer separations, and both excitonic and nonexcitonic NRET at shorter distances. In close proximity, the NRET rate from the QDs into the RGO layer is higher than the QDs into the GO layer, which mostly results from the structural defects. However, at longer separations $(>5 \mathrm{~nm})$, rates are almost the same for both hybrid nanostructures. In addition, it is found that the reduction process directly affects the behavior of NRET rates for these structures. Furthermore, NRET efficiencies are calculated from the experimental data to be high around 97 and $89 \%$ for the closest separation of the QDRGO and the QD-GO pairs, respectively. In light of these results, we conclude that hybrid structures including acceptor GO or RGO layers together with donor QDs hold great promise for use in several optoelectronic applications.

\section{ASSOCIATED CONTENT}

\section{S Supporting Information}

Supplementary figure for AFM analysis of the sample with 5 $\mathrm{nm} \mathrm{HfO}_{2}$ layer on GO film. This material is available free of charge via the Internet at http://pubs.acs.org. 


\section{AUTHOR INFORMATION}

\section{Corresponding Author}

*E-mail: volkan@stanfordalumni.org, hvdemir@ntu.edu.sg. Tel.: +90312-290-1021.

\section{Author Contributions}

${ }^{\dagger}$ These authors contributed equally to this work (A.Y. and G.K.-D.).

\section{Notes}

The authors declare no competing financial interest.

\section{ACKNOWLEDGMENTS}

This work is supported in part by EU-FP7 Nanophotonics4Energy NoE, and TUBITAK EEEAG 109E002, 109E004, 110E010, and 110E217 and in part by NRF-CRP-6-2010-02 and NRF-RF-2009-09. H.V.D. acknowledges support from ESF-EURYI and TUBA-GEBIP. Authors would like to thank to Dr. Erman Bengu for the CVD facility at Department of Chemistry at Bilkent University.

\section{REFERENCES}

(1) Novoselov, K. S.; Geim, A. K.; Morozov, S. V; Jiang, D.; Zhang, Y.; Dubonos, S. V; Grigorieva, I. V; Firsov, A. A. Electric Field Effect in Atomically Thin Carbon Films. Science 2004, 306, 666-669.

(2) Mak, K. F.; Sfeir, M. Y.; Wu, Y.; Lui, C. H.; Misewich, J. A.; Heinz, T. F. Measurement of the Optical Conductivity of Graphene. Phys. Rev. Lett. 2008, 101, 196405.

(3) Nair, R. R.; Blake, P.; Grigorenko, A. N.; Novoselov, K. S.; Booth, T. J.; Stauber, T.; Peres, N. M. R.; Geim, A. K. Fine Structure Constant Defines Visual Transparency of Graphene. Science 2008, 320, 1308.

(4) Zhu, Y.; Murali, S.; Cai, W.; Li, X.; Suk, J. W.; Potts, J. R.; Ruoff, R. S. Graphene and Graphene Oxide: Synthesis, Properties and Applications. Adv. Mater. 2010, 22, 3906-3924.

(5) Mayorov, A. S.; Gorbachev, R. V; Morozov, S. V; Britnell, L.; Jalil, R.; Ponomarenko, L. A; Blake, P.; Novoselov, K. S.; Watanabe, K.; Taniguchi, T.; et al. Micrometer-Scale Ballistic Transport in Encapsulated Graphene at Room Temperature. Nano Lett. 2011, 11, 2396-2399.

(6) Bonaccorso, F.; Sun, Z.; Hasan, T.; Ferrari, A. C. Graphene Photonics and Optoelectronics. Nat. Photonics 2010, 4, 611-622.

(7) Chen, Z.; Berciaud, S.; Nuckolls, C.; Heinz, T. F.; Brus, L. E. Energy Transfer from Individual Semiconductor Nanocrystals to Graphene. ACS Nano 2010, 4, 2964-2968.

(8) Gaudreau, L.; Tielrooij, K. J.; Prawiroatmodjo, G. E. D. K.; Osmond, J.; de Abajo, F. J. G.; Koppens, F. H. L. Universal DistanceScaling of Nonradiative Energy Transfer to Graphene. Nano Lett. 2013, 13, 2030-2035.

(9) Valeur, B. Molecular Fluorescence: Principles and Applications; Wiley-VCH: Weinheim, 2002.

(10) Lakowicz, J. R. Principles of Fluorescence Spectroscopy; Springer: New York, 2006.

(11) Guo, C. X.; Yang, H. B.; Sheng, Z. M.; Lu, Z. S.; Song, Q. L.; Li, C. M. Layered Graphene/Quantum Dots for Photovoltaic Devices. Angew. Chem., Int. Ed. 2010, 49, 3014-3017.

(12) Lin, Y.; Zhang, K.; Chen, W.; Liu, Y.; Geng, Z.; Zeng, J.; Pan, N.; Yan, L.; Wang, X.; Hou, J. G. Dramatically Enhanced Photoresponse of Free Anchored CdSe Nanoparticles. ACS Nano 2010, 4, 3033-3038.

(13) Swathi, R. S.; Sebastian, K. L. Resonance Energy Transfer from a Dye Molecule to Graphene. J. Chem. Phys. 2008, 129, 054703.

(14) Swathi, R. S.; Sebastian, K. L. Long Range Resonance Energy Transfer from a Dye Molecule to Graphene has (Distance)(-4) Dependence. J. Chem. Phys. 2009, 130, 086101.

(15) Konstantatos, G.; Badioli, M.; Gaudreau, L.; Osmond, J.; Bernechea, M.; Garcia de Arquer, F. P.; Gatti, F.; Koppens, F. H. L. Hybrid Graphene-Quantum Dot Phototransistors with Ultrahigh Gain. Nat. Nanotechnol. 2012, 7, 363-368.
(16) Loh, K. P.; Bao, Q.; Eda, G.; Chhowalla, M. Graphene Oxide as a Chemically Tunable Platform for Optical Applications. Nat. Chem. 2010, 2, 1015-1024.

(17) Park, S.; Ruoff, R. S. Chemical Methods for the Production of Graphenes. Nat. Nanotechnol. 2009, 4, 217-224.

(18) Kim, J.; Cote, L. J.; Kim, F.; Huang, J. Visualizing Graphene Based Sheets by Fluorescence Quenching Microscopy. J. Am. Chem. Soc. 2010, 132, 260-267.

(19) Morales-Narváez, E.; Pérez-López, B.; Pires, L. B.; Merkoçi, A. Simple Förster Resonance Energy Transfer Evidence for the Ultrahigh Quantum Dot Quenching Efficiency by Graphene Oxide Compared to Other Carbon Structures. Carbon 2012, 50, 2987-2993.

(20) Forster, T. Zwischenmolekulare Energiewanderung und Fluoreszenz. Annu. Phys. 1948, 437, 55-75.

(21) Kagan, C.; Murray, C.; Bawendi, M. Long-Range Resonance Transfer of Electronic Excitations in Close-Packed CdSe QuantumDot Solids. Phys. Rev. B 1996, 54, 8633-8643.

(22) Calizo, I.; Ghosh, S.; Bao, W.; Miao, F.; Ning Lau, C.; Balandin, A. A. Raman Nanometrology of Graphene: Temperature and Substrate Effects. Solid State Commun. 2009, 149, 1132-1135.

(23) Kudin, K. N.; Ozbas, B.; Schniepp, H. C.; Prud'homme, R. K.; Aksay, I. A; Car, R. Raman Spectra of Graphite Oxide and Functionalized Graphene Sheets. Nano Lett. 2008, 8, 36-41.

(24) Moon, I. K.; Lee, J.; Ruoff, R. S.; Lee, H. Reduced Graphene Oxide by Chemical Graphitization. Nat. Commun. 2010, 73, 1-6.

(25) Yang, D.; Velamakanni, A.; Bozoklu, G.; Park, S.; Stoller, M.; Piner, R. D.; Stankovich, S.; Jung, I.; Field, D. A.; Ventrice, C. A.; et al. Chemical Analysis of Graphene Oxide Films After Heat and Chemical Treatments by X-ray Photoelectron and Micro-Raman Spectroscopy. Carbon 2009, 47, 145-152.

(26) Shen, J.; Hu, Y.; Shi, M.; Lu, X.; Qin, C.; Li, C.; Ye, M. Fast and Facile Preparation of Graphene Oxide and Reduced Graphene Oxide Nanoplatelets. Chem. Mater. 2009, 21, 3514-3520.

(27) Lambert, T. N.; Luhrs, C. C.; Chavez, C. A.; Wakeland, S.; Brumbach, M. T.; Alam, T. M. Graphite Oxide as a Precursor for the Synthesis of Disordered Graphenes Using the Aerosol-ThroughPlasma Method. Carbon 2010, 48, 4081-4089.

(28) Sutar, D. S.; Narayanam, P. K.; Singh, G.; Botcha, V. D.; Talwar, S. S.; Srinivasa, R. S.; Major, S. S. Spectroscopic Studies of Large Sheets of Graphene Oxide and Reduced Graphene Oxide Monolayers Prepared by Langmuir-Blodgett Technique. Thin Solid Films 2012, 520, 5991-5996.

(29) Pimenta, M. A.; Dresselhaus, G.; Dresselhaus, M. S.; Cançado, L. G.; Jorio, A.; Saito, R. Studying Disorder in Graphite-Based Systems by Raman Spectroscopy. Phys. Chem. Chem. Phys. 2007, 9, 1276-1291.

(30) Zhou, Y.; Bao, Q.; Tang, L. A. L.; Zhong, Y.; Loh, K. P. Hydrothermal Dehydration for the "Green" Reduction of Exfoliated Graphene Oxide to Graphene and Demonstration of Tunable Optical Limiting Properties. Chem. Mater. 2009, 21, 2950-2956.

(31) Paredes, J. I.; Villar-Rodil, S.; Solís-Fernández, P.; MartínezAlonso, A.; Tascón, J. M. D. Atomic Force and Scanning Tunneling Microscopy Imaging of Graphene Nanosheets Derived from Graphite Oxide. Langmuir 2009, 25, 5957-5968.

(32) Tung, V. C.; Allen, M. J.; Yang, Y.; Kaner, R. B. HighThroughput Solution Processing of Large-Scale Graphene. Nat. Nanotechnol. 2009, 4, 25-29.

(33) Fan, Z.; Wang, K.; Wei, T.; Yan, J.; Song, L.; Shao, B. An Environmentally Friendly and Efficient Route for the Reduction of Graphene Oxide by Aluminum Powder. Carbon 2010, 48, 1686-1689.

(34) Pei, S.; Cheng, H. M. The Reduction of Graphene Oxide. Carbon 2012, 50, 3210-3228.

(35) Zangmeister, C. D. Preparation and Evaluation of Graphite Oxide Reduced at $220{ }^{\circ} \mathrm{C}$. Chem. Mater. 2010, 22, 5625-5629.

(36) Akhavan, O. The Effect of Heat Treatment on Formation of Graphene Thin Films from Graphene Oxide Nanosheets. Carbon 2010, 48, 509-519.

(37) Singh, D. K.; Iyer, P. K.; Giri, P. K. Role of Molecular Interactions and Structural Defects in the Efficient Fluorescence Quenching by Carbon Nanotubes. Carbon 2012, 50, 4495-4505. 
(38) Dong, H.; Gao, W.; Yan, F.; Ji, H.; Ju, H. Fluorescence Resonance Energy Transfer between Quantum Dots and Graphene Oxide for Sensing Biomolecules. Anal. Chem. 2010, 82, 5511-5517. 